\title{
A CYTOGENETIC SURVEY OF 14,835 CONSECUTIVE LIVEBORNS
}

\author{
Tohru Maeda, ${ }^{1}$ Michiko OHno, ${ }^{2}$ Akira Matsunobu, ${ }^{3}$ \\ Kyoko Yoshinara, ${ }^{3}$ and Noriko YABE ${ }^{4}$ \\ ${ }^{1}$ Kitasato University School of Nursing, \\ ${ }^{2}$ Cytogenetic Laboratory, Kitasato University Hospital, \\ ${ }^{3}$ Department of Obstetrics and Gynecology, Kitasato University School of Medicine, and \\ ${ }^{4}$ Department of Chinical Pathology, Kitasato University School of Medicine, \\ Sagamihara, Kanagawa 228, Japan
}

Summary The results of chromosome studies on cultured umbilical cord blood lymphocytes from a consecutive series of 14,835 liveborn infants (7,608 males and 7,227 females) are described. Ninety-three infants (6.27 per 1,000$)$ had a major chromosome abnormality. Of these, thirtyone infants $(2.09$ per 1,000) had sex chromosome abnormalities. Seven male infants had a 47,XXY karyotype, five had a 47,XYY karyotype, and three were mosaics. One male had a ring $Y$ chromosome in all cells examined. A pericentric inversion of the $Y$ chromosome was found in one case. Seven female infants had a $47, \mathrm{XXX}$ karyotype, one had a $45, \mathrm{X}$ karyotype and six were mosaics. Sixty-two infants $(4.18$ per 1,000$)$ had autosomal abnormalities. There were twenty-one infants with trisomy 21 including one mosaic, six infants with trisomy 18, and two infants with trisomy 13 of a Robertsonian translocation type. Three infants had an unbalanced derivative chromosome resulting from a parental reciprocal translocation. Two infants with a partial monosomy of chromosome 13 were detected. There were four infants carrying an additional small marker chromosome. Twenty-four infants $(1.62$ per 1,000$)$ had a balanced structural rearrangement of the autosomes; eleven with a Robertsonian translocation, eleven with a reciprocal translocation, and two with a pericentric inversion. The incidence of each type of major chromosome abnormality in this study was quite similar to that obtained from previous newborn surveys.

Key Words chromosome abnormality, cytogenetic survey, newborn population

Received December 21, 1989: revised version received February 7, 1991; Accepted February 8, 1991, 


\section{INTRODUCTION}

In 1978 we presented a preliminary report of a cytogenetic survey on 2,626 consecutive liveborn infants born during a period from December 1, 1975 to November 30, 1977 (Maeda et al., 1978). The survey was continued until December 31,1983 in the same manner, and for the following 3 years until December 31, 1986 using a G-banding method for chromosome analysis. In this report, we present the incidence and type of major chromosome abnormalities in 14,835 consecutive liveborn infants which were collected in one hospital during a period of 11 years and 1 month, and compare them with other livenorn surveys in the literature.

\section{MATERIALS AND METHODS}

Umbilical cord blood sampling, cell culture and chromosome analysis using conventional methods have been described previously (Maeda et al., 1978). During the first part of the survey, five metaphase cells were analyzed for each individual. When any chromosome abnormality was found during analysis of conventionally stained preparations, special staining methods including Q-banding and/or G-banding were used to identify the abnormal chromosomes. Slides from each infant born during last 3 years of the survey were stained with Giemsa after treatment of trypsin. During the latter part of the survey, the chromosomes of two cells from each infant were counted and fully analyzed under microscopy. The number of aralyzed metaphases was increased when mosaicism was suspected. When a balanced or an unbalanced structural rearrangement of the chromosomes was detected in an infant, blood samples were collected from both parents and their karyotypes were examined.

A total of 16,635 infants were born alive in the Kitasato University Hospital during a period from December 1, 1975 to December 31, 1986. Cytogenetic analyses were possible in 15,285 out of the 15,750 infants available for blood culture. During the period of investigation, 450 infants were born alive after prenatal karyotyping performed because of an increased risk of chromosomal aberration. The indications for prenatal karyotyping included advanced maternal age (320 cases), parental translocation (12 cases), previous child with Down's syndrome (30 cases) and miscellaneous ( 88 cases). Out of 12 pregnancies in which one parent had a known balanced translocation, 7 fetuses showed an identical translocation. All of these 450 infants were excluded from the study population in order to minimize possible selection bias.

The cytogenetic results obtained from a total of 14,835 infants consisting of 7,608 males and 7,227 females are presented. During the first period of investigation from 1975 to 1983 , a total of 10,801 infants (5,512 males and 5,289 females) were studied, and 4,034 infants (2,096 males and 1,938 females) were karyotyped 
Table 1. Anamnestic and clinical data of the study population (December 1, 1975 to December .31, 1986).

\begin{tabular}{lc}
\hline Total livebirths & 16635 \\
Blood samples obtained & 15750 \\
Unsuccessful cultures & 465 \\
Infants born after prenatal diagnosis & 450 \\
Infants successfully karyotyped a & 14835 \\
$\quad$ Males & 7608 \\
$\quad$ Females & 7227 \\
$\quad$ Sex ratio & 1.05 \\
Mean maternal age (years) & $29.7 \pm 3.9$ \\
Mean gestational age (weeks) & $38.4 \pm 1.8$ \\
Mean birth weight (g) & \\
$\quad$ Male & $3002 \pm 442$ \\
$\quad$ Fenale & $2909 \pm 421$ \\
Mean birth length (cm) & \\
Male & $48.4 \pm 2.4$ \\
$\quad$ Female & $47.8 \pm 2.4$ \\
\hline
\end{tabular}

a Infants with a prenatal diagnosis are excluded.

during the last 3 years from 1984 to 1986 . Approximately three-quarters of the participating mothers (11,353 of 14,835 mothers) live in Kanagawa prefecture. During the study period, there were $1,052,880$ live births in this area, thus $1.1 \%$ of these births were included in this survey. Some anamnestic and clinical data of the study population are listed in Table 1.

\section{RESULTS}

Of the 14,835 infants in whom a successful karyotype analysis was possible, 93 infants $(6.27$ per 1,000$)$ had a major chromosome abnormality. Four infants with an additional minute chromosome were included in this total. We decided to consider a pericentric inversion of chromosome 9 as normal variant, and it is not included in the total of 93 major abnormalities.

1. Sex chromosome abnormalities in phenotypic males (Table 2)

Among the 7,608 male infants in whom a successful karyotype analysis was possible, 17 infants $(2.23$ per 1,000$)$ had sex chromosome abnormalities. Seven $(0.92$ per 1,000$)$ had a $47, X X Y$ karyotype and five $(0.66$ per 1,000$)$ had a $47, X Y Y$ karyotype. Three infants with mosaic sex chromosomes $(46, \mathrm{XY} / 47, \mathrm{XXY}, 46, \mathrm{XY})$ $47, X Y Y$, and $45, X / 46, X Y / 47, X Y Y$ ) were detected. Pericentric inversion of the $Y$ chromosome was found in one case (356/76) and one infant (169/85) had a ring 
Table 2. Male infants with sex chromosome abnormalities.

\begin{tabular}{|c|c|c|c|c|c|c|c|}
\hline $\begin{array}{l}\text { Newborn } \\
\text { number }\end{array}$ & Karyotype & $\begin{array}{l}\text { Parent } \\
\text { mother }\end{array}$ & & $\begin{array}{l}\text { Gesta- } \\
\text { tion } \\
\text { (weeks) }\end{array}$ & $\begin{array}{l}\text { Birth } \\
\text { weight } \\
\text { (g) }\end{array}$ & $\begin{array}{l}\text { Birth } \\
\text { length } \\
(\mathrm{cm})\end{array}$ & Comments \\
\hline $163 / 79$ & $47, X X Y$ & 25 & 25 & 40 & 2505 & 46.0 & No physical abnormality \\
\hline $1118 / 80$ & $47, \mathrm{XXY}$ & 33 & 33 & 39 & 3290 & 50.0 & No physical abnormality \\
\hline $16 / 81$ & $47, X X Y$ & 26 & 30 & 38 & 2565 & 46.3 & No physical abnormality \\
\hline $897 / 82$ & $47, X X Y$ & 33 & 37 & 38 & 3630 & 49.0 & No physical abnormality \\
\hline $708 / 85$ & $47, \mathrm{XXY}$ & 31 & 35 & 38 & 3440 & 49.5 & No physical abnormality \\
\hline $1553 / 85$ & $47, \mathrm{XXY}$ & 29 & - & 35 & 3003 & 48.6 & No physical abnormality \\
\hline $1142 / 86$ & $47, X X Y$ & 30 & 38 & 39 & 3152 & 48.0 & No physical abnormality \\
\hline $1088 / 78$ & $46, X Y / 47, X X Y$ & 23 & 33 & 40 & 3218 & 51.0 & No physical abnormality \\
\hline $6 / 77$ & $47, X Y Y$ & 37 & 36 & 38 & 2682 & 48.0 & No physical abnormality \\
\hline $203 / 77$ & $47, X Y Y$ & 40 & 37 & 36 & 2417 & 45.0 & No physical abnormality \\
\hline $924 / 79$ & $47, X Y Y$ & 25 & 29 & 40 & 2410 & 48.0 & No physical abnormality \\
\hline $272 / 82$ & $47, X Y Y$ & 35 & 35 & 39 & 3012 & 47.8 & No physical abnormality \\
\hline $1431 / 86$ & $47, X Y Y$ & 26 & - & 39 & 2904 & 49.5 & No physical abnormality \\
\hline $394 / 76$ & $46, X Y / 47, X Y Y$ & 29 & 29 & 38 & 2927 & 48.0 & No physical abnormality \\
\hline $389 / 76$ & $45, X / 46, X Y / 47, X Y Y$ & 32 & 35 & 38 & 2761 & 47.5 & No physical abnormality, twin \\
\hline $356 / 76$ & $46, X, \operatorname{inv}(Y)$ & 28 & - & 38 & 3265 & 49.7 & No physical abnormality \\
\hline $169 / 85$ & $46, X, r(X)$ & 29 & - & 38 & 2935 & 49.5 & No physical abnormality \\
\hline
\end{tabular}

Y chromosome without mosaicism. In all infants no physical abnormality was found at the time of birth. The incidences of male infants with a sex chromosome abnormality during the two periods, $1975-1983$ and 1984-1986, were 2.18 and 2.39 per 1,000 , respectively (Table 7 ).

\section{Sex chromosome abnormalities in phenotypic females (Table 3)}

In the 7,227 female infants in whom a successful karyotype analysis was possible, $14(1.94$ per 1,000$)$ had a sex chromosome abnormality. One infant with a $45, \mathrm{X}$ chromosome complement $(190 / 86)$ had evidence of intrauterine growth retardation and pedal lymphedema, and expired 2 days after birth. Seven infants (0.97 per 1,000$)$ had a $47, X X X$ karyotype. Six infants were mosaics; one was $45, \mathrm{X} / 46, \mathrm{XX}$, one was $46, \mathrm{XX} / 47, \mathrm{XXX}$, one was $45, \mathrm{X} / 46, \mathrm{XY} / 47, \mathrm{XYY}$, two were $45, X / 47, X X X$ and one was $45, X / 46, X, \operatorname{idic}(X)(q 27)$. One of these infants with a $45, \mathrm{X} / 47, \mathrm{XXX}$ karyotype $(1008 / 80)$ had a webbed neck at the time of birth, while the remaining five infants showed no physical abnormality. Cases 388/76 and $389 / 76$ (Table 2) were twins with an apparent opposite sex. The incidence of female infants with a sex chromosome abnormality during the first period $(1.70$ per 1,000$)$ 
Table 3. Female infants with sex chromosome abnormalities.

\begin{tabular}{|c|c|c|c|c|c|c|c|}
\hline $\begin{array}{l}\text { Newborn } \\
\text { number }\end{array}$ & Karyotype & $\begin{array}{l}\text { Parental } \\
\text { mother }\end{array}$ & $\begin{array}{l}1 \text { age } \\
\text { father }\end{array}$ & $\begin{array}{l}\text { Gesta- } \\
\text { tion } \\
\text { (weeks) }\end{array}$ & $\begin{array}{l}\text { Birth } \\
\text { weight } \\
\text { (g) }\end{array}$ & $\begin{array}{l}\text { Birth } \\
\text { length } \\
(\mathrm{cm})\end{array}$ & Conments \\
\hline $180 / 86$ & $45, X$ & 38 & - & 38 & 2102 & 45.5 & Died at 2 days of life \\
\hline $1415 / 76$ & $45, X / 46, X X$ & 27 & 31 & 39 & 3130 & 47.0 & No physical abnormality \\
\hline $327 / 76$ & $45, X / 47, X X X$ & 28 & 37 & 38 & 2643 & 47.0 & No physical abnormality \\
\hline $1008 / 80$ & $45, X / 47, X X X$ & 24 & 29 & 38 & 2327 & 44.5 & Webbed neck \\
\hline $388 / 76$ & $45, X / 46, X Y / 47, X Y Y$ & 32 & 35 & 38 & 2761 & 48.0 & No physical abnormality, twin \\
\hline $1253 / 85$ & $45, X / 46, X, \operatorname{idic}(X)(\mathrm{q} 27)$ & 24 & - & 38 & 3182 & 46.5 & No physical abnormality \\
\hline $1224 / 76$ & $47, \mathrm{XXX}$ & 25 & 25 & 39 & 2363 & 45.5 & No physical abnormality \\
\hline $524 / 78$ & $47, X X X$ & 30 & 33 & 38 & 2715 & 45.0 & No physical abnormality \\
\hline $1107 / 79$ & $47, X X X$ & 28 & 34 & 39 & 2470 & 45.0 & No physical abnormality \\
\hline $192 / 80$ & $47, \mathrm{XXX}$ & 39 & 42 & 38 & 3089 & 49.0 & No physical abnormality \\
\hline $172 / 84$ & $47, X 88$ & 23 & - & 39 & 2040 & 45.0 & Ko physical abnormality \\
\hline $1148 / 84$ & $47, \mathrm{XXX}$ & 35 & - & 38 & 2520 & 46.5 & No physical abnormality \\
\hline $965 / 86$ & $47, X X X$ & 29 & - & 37 & 2265 & 46.6 & No physical abnormality \\
\hline $1263 / 82$ & $46, X X / 47, X X X$ & 38 & - & 40 & 2830 & 48.0 & No physical abnormality \\
\hline
\end{tabular}

and second period (2.58 per 1,000) was not significantly different (Table 7).

\section{Autosomal trisomy (Table 4)}

Twenty-one infants (1.42 per 1,000) had a karyotype with trisomy 21 including one mosaic (1031/83). The incidence of infants with trisomy 21 during the first period (1.48 per 1,000) was not different from that of the second period (1.24 per 1,000 ) (Table 8). All of the 21 infants had the clinical features of Down's syndrome. Six infants $(0.40$ per 1,000$)$ with an additional chromosome 18 showed the clinical features of Edwards' syndrome and all died soon after birth. Three infants $(0.20$ per 1,000$)$ had an additional small marker chromosome in all cells examined, and one was a $46, \mathrm{XY} / 47, \mathrm{XY}$, + mar mosaic. In these cases the origin of the marker chromosome was not identified.

\section{Balanced structural rearrangements of autosomes (Table 5)}

Twenty-four infants $(1.62$ per 1,000$)$ had a balanced structural rearrangements of autosomes. Eleven $(0.74$ per 1,000$)$ were a Robertsonian translocation, $11(0.74$ per 1,000$)$ were a reciprocal translocation, and two $(0.13$ per 1,000$)$ were a pericentric inversion. There was no significant difference between the incidences of balanced Robertsonian translocations during the first period $(0.65$ per 1,000$)$ and the second period $(0.99$ per 1,000$)$. The incidence of infants with a balanced reciprocal translocation was 0.74 per 1,000 in both the first and second periods. Nine of the 11 
Table 4. Infants with an abnormal number of autosomes.

\begin{tabular}{|c|c|c|c|c|c|c|c|}
\hline $\begin{array}{l}\text { Newborn } \\
\text { number }\end{array}$ & Karyotype & $\begin{array}{l}\text { Parents } \\
\text { mother }\end{array}$ & $\begin{array}{l}\text { age } \\
\text { ather }\end{array}$ & $\begin{array}{l}\text { Gesta- } \\
\text { tion } \\
\text { (weeks) }\end{array}$ & $\begin{array}{l}\text { Birth } \\
\text { weight } \\
\text { (g) }\end{array}$ & $\begin{array}{l}\text { Birth } \\
\text { length } \\
\text { (cm) }\end{array}$ & Comments \\
\hline $316 / 77$ & $47, X Y,+21$ & 27 & 27 & 39 & 2650 & 44.0 & Down's syndrome \\
\hline $699 / 77$ & $47, X X,+21$ & 25 & 27 & 39 & 2147 & 45.5 & Down's syndrome \\
\hline $1243 / 77$ & $47,8 X,+21$ & 21 & 32 & 39 & 1945 & 45.0 & Down's syndrome \\
\hline $391 / 78$ & $47, X X,+21$ & 35 & - & 38 & 3381 & - & Down's syndrome \\
\hline $650 / 79$ & $47, X X,+21$ & 25 & - & 39 & 2487 & 46.0 & Down's syndrome \\
\hline $792 / 79$ & $47, X Y,+21$ & 30 & - & 38 & 3440 & 48.0 & Down's syndrome \\
\hline $972 / 79$ & $47, X Y,+21$ & 23 & 26 & 34 & 1500 & 40.0 & Down's syndrome \\
\hline $475 / 80$ & $47, X X,+21$ & 35 & 37 & 38 & 2910 & 45.8 & Down's syndrome \\
\hline $478 / 80$ & $47, X Y,+21$ & 29 & 28 & 35 & 2500 & 46.0 & Down's syndrome \\
\hline $38 / 81$ & $47, X X,+21$ & 27 & - & 37 & 2795 & 48.0 & Down's syndrome \\
\hline $1071 / 81$ & $47, X Y,+21$ & 36 & - & 38 & 3020 & 45.1 & Down's syndrome \\
\hline $242 / 82$ & $47, X X,+21$ & 31 & 34 & 40 & 2881 & 47.5 & Down's syndrome \\
\hline $577 / 82$ & $47, X Y,+21$ & 31 & - & 38 & 3380 & 47.0 & Down's syndrome \\
\hline $420 / 83$ & $47,8 Y,+21$ & 36 & - & 39 & 3551 & 49.5 & Down's syndrome \\
\hline $1520 / 83$ & $47, X Y,+21$ & 40 & - & 39 & 2875 & 45.5 & Down's syndrome \\
\hline $661 / 84$ & $47, X Y,+21$ & 28 & 30 & 28 & 1568 & 41.5 & Down's syndrome, died at 56 days \\
\hline $1190 / 84$ & $47, X Y,+21$ & 26 & 31 & 38 & 2282 & 46.0 & Down's syndrome \\
\hline $1351 / 84$ & $47,8 Y,+21$ & 38 & 36 & 37 & 2231 & 44.2 & Down's syndrome \\
\hline $397 / 85$ & $47, X Y,+21$ & 35 & 37 & 39 & 3142 & 49.5 & Down's syndrome \\
\hline $1454 / 85$ & $47, X Y,+21$ & 35 & 42 & 37 & 2415 & 44.5 & Down's syndrome \\
\hline $1031 / 83$ & $46, X X / 47, X X,+21$ & 29 & - & 38 & 3316 & 49.5 & Down's syndrome \\
\hline $1202 / 79$ & $47, X X,+18$ & 30 & 31 & 32 & 768 & 34.5 & Edwards' syndrome, died at 3 days \\
\hline $522 / 80$ & $47, \mathrm{XX},+18$ & 26 & 28 & 40 & 1927 & 41.5 & Edwards' syndrone, died at 10 days \\
\hline $555 / 80$ & $47, X Y,+18$ & 31 & 35 & 37 & 1890 & 40.0 & Edwards' syndrome, died at 4 days \\
\hline $1336 / 83^{\circ}$ & $47, X X,+18$ & 31 & - & 39 & 1960 & 42.0 & Edwards' syndrome, died at 120 days \\
\hline $347 / 84$ & $47, X X,+18$ & 28 & 33 & 38 & 1906 & 41.5 & Edwards' syndrome, died at 72 days \\
\hline $455 / 86$ & $47, X Y,+18$ & 35 & - & 36 & $\Xi 152$ & 37.5 & Edwards' sydrome, died at I day \\
\hline $700 / 76$ & $47, \mathrm{XY},+\operatorname{mar}$ & 38 & 一 & 37 & 3050 & 48.5 & No physical abnornality \\
\hline $1032 / 83$ & $47, X Y,+\operatorname{mar}$ & 33 & 33 & 39 & 2739 & 50.0 & No physical abnormality \\
\hline $947 / 86$ & $47, \mathrm{XY},+\operatorname{mar}$ & 34 & - & 40 & 3356 & 49.0 & No physical abnormality \\
\hline $345 / 84$ & $46, X Y / 47, X Y,+m a r$ & 34 & 38 & 38 & 3090 & 49.0 & No physical abnormality \\
\hline
\end{tabular}

Robertsonian translocations identified were $t(13 q 14 q)$, one was $t(14 q 22 q)$, and the remaining one was $t(21 q 22 q)$. Nine of the 11 Robertsonian translocations were familial; seven were inherited from the mother, and two from the father. In one family (765/85), both the mother and father had a normal karyotype. In one 
Table 5. Cytogenetic and clinical data from infants with a balanced structural rearrangement of autosomes.

\begin{tabular}{|c|c|c|c|c|c|c|c|c|}
\hline \multirow{2}{*}{$\begin{array}{l}\text { Newborn } \\
\text { number }\end{array}$} & \multirow{2}{*}{$\begin{array}{l}\text { Karyotype } \\
45, X Y, t(13 q 14 q)\end{array}$} & \multicolumn{2}{|c|}{$\begin{array}{l}\text { Parental } \\
\text { karyotype } \\
\text { mother father }\end{array}$} & \multicolumn{2}{|c|}{$\begin{array}{l}\text { Parental } \\
\text { age } \\
\text { mother father }\end{array}$} & \multirow{2}{*}{$\begin{array}{l}\begin{array}{l}\text { Gesta- } \\
\text { tion } \\
\text { (weeks) }\end{array} \\
\frac{39}{39}\end{array}$} & \multirow{2}{*}{$\begin{array}{l}\begin{array}{l}\text { Birth } \\
\text { weight } \\
(\mathrm{g})\end{array} \\
2900\end{array}$} & \multirow{2}{*}{$\begin{array}{c}\begin{array}{c}\text { Birth } \\
\text { length } \\
(\mathrm{cm})\end{array} \\
49.0\end{array}$} \\
\hline & & $N$ & $\mathrm{Ab}$ & 26 & 35 & & & \\
\hline $1039 / 76$ & $45, X Y, t(13 q 14 q)$ & $\mathrm{Ab}$ & $\mathbb{N}$ & 31 & 33 & 38 & 2900 & 49.0 \\
\hline $281 / 77$ & $45, X X, t(13 q 14 q)$ & $A b$ & $N$ & 26 & 30 & 39 & 2963 & 49.0 \\
\hline $168 / 78$ & $45, X X, t(13 q 14 q)$ & $\mathrm{Ab}$ & $\mathrm{N}$ & 28 & 29 & 37 & 2633 & 47.5 \\
\hline $169 / 78$ & $45, \mathrm{XX}, \mathrm{t}(13 \mathrm{q} 14 \mathrm{q})$ & $A b$ & $N$ & 28 & 29 & 37 & 2205 & 46.0 \\
\hline $504 / 80$ & $45, X X, t(13 q 14 q)$ & $N$ & $\mathrm{Ab}$ & 26 & - & 39 & 3425 & 50.5 \\
\hline $51 / 85$ & $45, X Y, t(13 q 14 q)$ & - & - & 32 & - & 38 & 2960 & 49.2 \\
\hline $765 / 85$ & $45, X Y, t(13 q 14 q)$ & $\mathbb{N}$ & $\mathbb{N}$ & 28 & - & 39 & 2780 & 47.0 \\
\hline $1251 / 85$ & $45, X Y, t(13 q 14 q)$ & $\mathrm{Ab}$ & $N$ & 27 & - & 39 & 2446 & 45.0 \\
\hline $1242 / 77$ & $45, X X, t(14 q 22 q)$ & $\mathrm{Ab}$ & N & 26 & 28 & 39 & 3778 & 50.5 \\
\hline $1324 / 85$ & $45, X Y, t(21 q 22 q)$ & $A b$ & N & 26 & - & 36 & 2343 & 45.8 \\
\hline $209 / 78$ & $46, \mathrm{XY}, \mathrm{t}(1 ; 5)(\mathrm{p} 22 ; \mathrm{q} 33)$ & $A b$ & $N$ & 26 & 35 & 38 & 2860 & 48.0 \\
\hline $812 / 79$ & $46, \mathrm{XX}, \mathrm{t}(10 ; 17)(\mathrm{q} 22 ; \mathrm{q} 25)$ & - & - & 30 & 32 & 37 & 4131 & 51.0 \\
\hline $180 / 80$ & $46, \mathrm{XX}, \mathrm{t}(2 ; 5)(\mathrm{q} 14 ; \mathrm{q14})$ & $N$ & $\mathrm{Ab}$ & 31 & 31 & 39 & 3139 & 48.5 \\
\hline $327 / 80$ & $46, x Y, t(1 ; 18)(q 21 ; q 21)$ & $A B$ & $N$ & 33 & 32 & 27 & 1110 & - \\
\hline $1156 / 80$ & $46, \mathrm{XX}, \mathrm{t}(\mathrm{Cp}+; \mathrm{Dq}-)$ & $\mathrm{N}$ & $\mathrm{N}$ & 27 & 31 & 38 & 2757 & 46.0 \\
\hline $503 / 81$ & $46, \mathrm{XY}, \mathrm{t}(16 ; 17)(\mathrm{p} 11 ; q 12)$ & $\mathrm{Ab}$ & $N$ & 32 & 31 & 38 & 2800 & 47.5 \\
\hline $809 / 82$ & $46, X X, t(9 ; 10)(q 22 ; q 25)$ & $A b$ & $\mathbb{N}$ & 34 & 34 & 37 & 3191 & 48.5 \\
\hline $79 / 83$ & $46, X Y, t(2 ; 5)(q 14 ; q 14)$ & N & $\mathrm{Ab}$ & 34 & 34 & 39 & 3369 & 49.8 \\
\hline $505 / 84$ & $46, X Y, t(5 ; 16)(q 13 ; q 24)$ & $N$ & $\mathrm{~N}$ & 32 & 35 & 39 & 2815 & 48.0 \\
\hline $143 / 85$ & $46, \mathrm{XY}, \mathrm{t}(5 ; 18)(\mathrm{q} 31 ; \mathrm{pll})$ & $A b$ & $\mathrm{~N}$ & 31 & 31 & 35 & 2067 & 42.5 \\
\hline $173 / 86$ & $46, X Y, t(11 ; 22)(q 23 ; q 11)$ & - & - & 32 & 37 & 37 & 3031 & 48.2 \\
\hline $1444 / 82$ & $46, \mathrm{XX}, \operatorname{inv}(3)(\mathrm{p} 12 ; \mathrm{q} 25)$ & $\mathrm{Ab}$ & $\mathrm{N}$ & 26 & 38 & 39 & 3504 & 50.7 \\
\hline $804 / 83$ & $46, X X, \operatorname{inv}(2)(p 23 ; q 21)$ & $N$ & $\mathrm{Ab}$ & 38 & - & 39 & 3013 & 47.0 \\
\hline
\end{tabular}

$\mathrm{N}$, normal; $\mathrm{Ab}$, abnormal.

family (51/85), the parents were not available for study. All of the 24 infants showed no physical abnormality at the time of birth. In one infant (505/84), a balanced reciprocal translocation found with the use of banding techniques might have easily been interpreted as normal without banding. Seven of the 11 reciprocal translocations were familial; five were inherited from the mother, and two from the father. Two cases $(180 / 80$ and $79 / 83)$ with a $t(2 ; 5)$ were siblings. In two families 
(1156/80 and 505/84), both parents had a normal karyotype, and in two other families $(812 / 79$ and $173 / 86)$ blood samples were not available for study. In two cases with a pericentric inversion, one was inherited from the mother, and the other from the father.

\section{Unbalanced structural rearrangements of autosomes (Tables $6 \mathrm{~A}$ and $6 \mathrm{~B}$ )}

Seven infants $(0.47$ per 1,000$)$ had an unbalanced structural rearrangement of autosomes. Of these, five infants $(0.46$ per 1,000$)$ were detected during the first period, and two $(0.50$ per 1,000$)$ were detected during the second period of the study. All seven infants died within one year after birth.

Case $346 / 76$, with a $46, X X,-14,+t(13 q 14 q)$ karyotype was born to a 28 -yearold mother at 32 weeks of gestation. Her clinical features included microphthalmos, sloping forehead, cleft lip and palate, micrognathia, cyanosis, tachypea and retraction and hexadactyly of the left foot. She expired $12 \mathrm{hr}$ after birth. Autopsy

Table 6A. Cytogenetic data from infants with an unbalanced structural rearrangement of the autosomes.

\begin{tabular}{rlcc}
\hline \multirow{2}{*}{$\begin{array}{l}\text { Newborn } \\
\text { number }\end{array}$} & Karyotype & \multicolumn{2}{c}{ Parental karyotypes } \\
\cline { 3 - 4 } & mother & father \\
\hline $346 / 76$ & $46, \mathrm{XX},-14,+\mathrm{t}(13 \mathrm{q} 14 \mathrm{q})$ & $46, \mathrm{XX}$ & $45, \mathrm{XY}, \mathrm{t}(13 \mathrm{q} 14 \mathrm{q})$ \\
$1471 / 83$ & $46, \mathrm{XX},-13,+t(13 \mathrm{q} 13 \mathrm{q})$ & - & - \\
$468 / 84$ & $46, \mathrm{XY}, \mathrm{r}(13)(\mathrm{p} 12 \mathrm{q} 34)$ & - & - \\
$605 / 78$ & $46, \mathrm{XY}, \operatorname{del}(13)(\mathrm{q} 22)$ & $46, \mathrm{XX}$ & $46, \mathrm{XY}$ \\
$51 / 79$ & $46, \mathrm{XY}, \operatorname{der}(17), \mathrm{t}(9 ; 17)(\mathrm{p} 11 ; \mathrm{p} 13)$ & $46, \mathrm{XX}$ & $46, \mathrm{XY}, \mathrm{t}(9 ; 17)(\mathrm{p} 11 ; \mathrm{p} 13)$ \\
$1066 / 84$ & $46, \mathrm{XX}, \operatorname{der}(4), \mathrm{t}(4 ; 9)(\mathrm{p} 15 ; \mathrm{p} 13)$ & $46, \mathrm{XX}, \mathrm{t}(4 ; 9)(\mathrm{p} 15 ; \mathrm{p} 13)$ & $46, \mathrm{XY}$ \\
$1163 / 79$ & $46, \mathrm{XX}, \operatorname{der}(9), \mathrm{t}(1 ; 9)(\mathrm{q} 41 ; \mathrm{p} 24)$ & $46, \mathrm{XX}$ & $46, \mathrm{XY}, \mathrm{t}(1 ; 9)(\mathrm{q} 41 ; \mathrm{p} 24)$ \\
\hline
\end{tabular}

Table 6B. Clinical data of infants with an unbalanced structural rearrangement of the autosomes.

\begin{tabular}{rlrllll}
\hline $\begin{array}{l}\text { Newborn } \\
\text { number }\end{array}$ & \multicolumn{2}{l}{$\begin{array}{l}\text { Parental age } \\
\text { mother father }\end{array}$} & $\begin{array}{l}\text { Gesta- Birth } \\
\text { tion } \\
\text { (weeks) } \\
\text { weight } \\
(\mathrm{g})\end{array}$ & $\begin{array}{l}\text { Birth } \\
\text { length } \\
\text { (cm) }\end{array}$ & Comments \\
\hline $346 / 76$ & 28 & 29 & 32 & 1280 & - & Patau's syndrome, died at 12 hours \\
$1471 / 83$ & 31 & 34 & 38 & 3142 & 49.0 & Patau's syndrome, died at 2 days \\
$468 / 84$ & 25 & 30 & 39 & 1450 & 33.0 & Multiple congenital anonalies, died at 1 day \\
$605 / 78$ & 29 & 33 & 31 & 925 & 32.0 & Multiple congenital anomalies, died at 1 day \\
$51 / 79$ & 26 & 28 & 38 & 1925 & 44.0 & Multiple congenital anomalies, died at 8 months \\
$1066 / 84$ & 30 & 27 & 40 & 1588 & 40.0 & Multiple congenital anomalies, died at 11 days \\
$1163 / 79$ & 27 & 24 & 38 & 2519 & 46.0 & Multiple congenital anomalies, died at 8 months \\
\hline
\end{tabular}


revealed coarctation of the aorta, PDA, and polysplenia. A chromosome study of both parents revealed a normal karyotype in the mother, and $45, \mathrm{XY},-13,-14$, $+t(13 q 14 q)$ carrier status in the father.

Case $1471 / 83$, with a $46, X X,-13,+t(13 q 13 q)$ karyotype, was born to a 31 year-old mother at 38 weeks of gestation. She had the clinical features associated with Patau's syndrome. The parents were not available for chromosome study.

Case 468/84, with a 46,XY,r(13) karyotype, was delivered by cesarean section at 39 weeks of gestation with a birthweight of $1,450 \mathrm{~g}$. He was apneic and flaccid, and died $1 \mathrm{hr}$ after birth. Anomalies included microcephaly, a broad nasal bridge,

Table 7. Comparison of sex chromosome abnormalities (rate/1000 in parentheses).

\begin{tabular}{|c|c|c|c|c|c|c|}
\hline & & \multicolumn{3}{|c|}{ Present study } & \multirow{2}{*}{$\begin{array}{l}7 \text { surveys } \\
\text { without } \\
\text { banding }{ }^{2}\end{array}$} & \multirow{2}{*}{$\begin{array}{l}3 \text { surveys } \\
\text { with } \\
\text { banding b }\end{array}$} \\
\hline & & $1975-1983$ & $1984-1986$ & combined & & \\
\hline \multicolumn{2}{|c|}{ Total population } & 10801 & 4034 & 14835 & 54749 & 6753 \\
\hline \multicolumn{2}{|c|}{ Total males } & 5512 & 2096 & 7608 & 34379 & 3520 \\
\hline \multicolumn{2}{|c|}{ Total females } & 5289 & 1938 & 7227 & 20370 & 3233 \\
\hline \multirow[t]{7}{*}{ Males } & $47, X X Y$ & $4(0.73)$ & $3(1.43)$ & $7(0.92)$ & $33(0.96)$ & $7(1.99)$ \\
\hline & $47, \mathrm{XXY}$, mosaics & $1(0.18)$ & - & $1(0.13)$ & $6(0.17)$ & $1(0.28)$ \\
\hline & $47, X Y Y$ & $4(0.73)$ & $1(0.48)$ & $5(0.66)$ & $28(0.81)$ & $7(1.99)$ \\
\hline & $47, \mathrm{XYY}$, mosaics & $2(0.36)$ & - & $2(0.26)$ & $7(0.20)$ & - \\
\hline & $46, X, \operatorname{inv}(Y)$ & $1(0.18)$ & - & $1(0.13)$ & $9(0.26)$ & $3(0.85)$ \\
\hline & Others & - & $1^{c}(0.48)$ & $1^{c}(0.13)$ & $3(0.09)$ & - \\
\hline & Total & $12(2.18)$ & $5(2.39)$ & $17(2.23)$ & $86(2.50)$ & $18(5.11)$ \\
\hline \multirow[t]{6}{*}{ Females } & $45, x$ & 一 & $1(0.52)$ & $1(0.14)$ & $2(0.10)$ & - \\
\hline & $45, X$, mosaics & $1(0.19)$ & $1(0.52)$ & $2(0.28)$ & $6(0.29)$ & - \\
\hline & $47, \mathrm{XXX}$ & $4(0.76)$ & $3(1.55)$ & $7(0.97)$ & $20(0.98)$ & $3(0.93)$ \\
\hline & $47, X X X$, mosaics & $3(0.57)$ & 一 & $3(0.42)$ & $4(0.20)$ & - \\
\hline & Others & $1^{d}(0.19)$ & - & $1^{d}(0.14)$ & - & - \\
\hline & Total & $9(1.70)$ & $5(2.58)$ & $14(1.94)$ & $32(1.57)$ & $3(0.93)$ \\
\hline \multicolumn{2}{|c|}{$\begin{array}{l}\text { Total sex chromosome } \\
\text { abnormalities }\end{array}$} & $21(1.94)$ & $10(2.48)$ & $31(2.09)$ & $118(2.16)$ & $21(3.11)$ \\
\hline
\end{tabular}

a Nielsen et al. (1975), Lin et al. (1976) 930 infants, Buckton et al. (1980) 3993 infants, Hansteen et al. (1982) 1830 infants, c $46, \mathrm{X}, \mathrm{r}(\mathrm{Y})$, d 45,X/46,XY/47,XYY. 
hypertelorism, low set ears, micrognathia, macroglossia, a short webbed neck, an imperforate anus, an absent left thumb, hypospadias, cryptorchidism and talipes equinovarus. Autopsy findings included aplasia of the falx cerebri, agenesis of the corpus callosum, hypoplastic optic nerve, dilated fourth ventricle, ventricular septal defect and accessory spleens..

Case 605/78, with a 46,XY,del(13)(q22) karyotype was born to 29-year-old mother at 31 weeks of gestation. He weighed $925 \mathrm{~g}$, and died $1 \mathrm{hr}$ after birth. There was a partial defect of the cranium, meningo-encephalocele, cleft palate, low-set malformed ears, micrognathia, hypoplastic thumbs, imperforate anus, single flexion crease of the 5th fingers, syndactyly of 4th and 5th toes and overlapping of second toes onto the big toes. Autopsy revealed a PDA, hypoplastic polycystic kidneys and a hypoplastic gallbladder.

Table 8. Comparison of autosomal abnormalities (rate/1000 in parentheses).

\begin{tabular}{|c|c|c|c|c|c|}
\hline & \multicolumn{3}{|c|}{ Present study } & \multirow{2}{*}{$\begin{array}{l}7 \text { surveys } \\
\text { wi.thout } \\
\text { banding }{ }^{\mathrm{a}}\end{array}$} & \multirow{2}{*}{$\begin{array}{l}5 \text { surveys } \\
\text { with } \\
\text { bandingb }\end{array}$} \\
\hline & $1975-1983$ & $1984-1986$ & combined & & \\
\hline Total population & 10801 & 4034 & 14835 & 54749 & 14076 \\
\hline+13 & - & - & - & $3(0.05)$ & - \\
\hline+18 & $4 .(0.37)$ & $2 \cdot(0.50)$ & $6(0.40)$ & $8(0.15)$ & $3(0.21)$ \\
\hline+21 & $16 \cdot(1.48)$ & $5(1.24)$ & $21(1.42)$ & $63(1.15)$ & $18(1.28)$ \\
\hline+ mar & $2 \cdot(0.19)$ & $1(0.25)$ & $3(0.20)$ & $12(0.22)$ & $5(0.36)$ \\
\hline +mar, mosaics & - & $1(0.25)$ & $1(0.07)$ & $5(0.09)$ & $5(0.36)$ \\
\hline Deletions & $1(0.09)$ & $1(0.25)$ & $2(0.13)$ & $5(0.09)$ & - \\
\hline Inversions & $2(0.19)$ & - & $2(0.13)$ & $7(0.13)$ & $6(0.43)$ \\
\hline \multicolumn{6}{|c|}{ Robertsonian translocations } \\
\hline balanced & $7(0.65)$ & $4(0.99)$ & $11(0.74)$ & $52(0.95)$ & $13(0.92)$ \\
\hline unbalanced & $2(0.19)$ & - & $2(0.13)$ & $2(0.04)$ & - \\
\hline \multicolumn{6}{|l|}{ Reciprocal translocations } \\
\hline balanced & $8(0.74)$ & $3(0.74)$ & $11(0.74)$ & $46(0.84)$ & $17(1.21)$ \\
\hline unbalanced & $2(0.19)$ & $1(0.25)$ & $3(0.20)$ & $1(0.02)$ & - \\
\hline Y/Autosome translocations & - & - & - & $6(0.11)$ & $1(0.07)$ \\
\hline Others & - & - & - & $2(0.04)$ & $3^{c}(0.21)$ \\
\hline $\begin{array}{l}\text { Total autosomal } \\
\text { abnormalities }\end{array}$ & $44(4.07)$ & $18(4.46)$ & $62(4.18)$ & $212(3.87)$ & $71(5.04)$ \\
\hline
\end{tabular}

a Nielsen et al. (1975), b Lin et al. (1976) 930 infants, Buckton et al. (1980) 3993 infants, Hansteen et al. (1982) 1830 infants, Nielsen et al. (1982) 3658 infants, Bratkowska et al. (1985) 3665 infants,

c One with mosaic trisomy 8 , two with duplication. 
Case $51 / 79$, with a $46, \mathrm{XY}, \operatorname{der}(17), \mathrm{t}(9 ; 17)(\mathrm{p} 11 ; \mathrm{q} 13)$ karyotype showed a characteristic phenotype of trisomy $9 \mathrm{p}$, including brachycephaly, short neck, micrognathia, a protruding forehead, hypertelorism, a prominent large nose, short philtrum, downward slanted corners of the mouth, low-set malformed ears, club feet, short fingers, clinodactyly and a single flexion crease of 5th finger. Computed tomography of the head revealed slightly dilated ventricles. At the age of 8 months, he was readmitted because of cardiac insufficiency and expired at 255 days after birth. Autopsy revealed hypoplasia of tricuspid and mitral valves, and a PDA.

Case 1066/84, with a 46,XX, $\operatorname{der}(4), t(4 ; 9)(\mathrm{p} 15 ; \mathrm{p} 13)$ karyotype had the following clinical features suggestive of trisomy $9 \mathrm{p}$ : hypertelorism, strabismus, antimongoloid slant of the palpebral fissures, a broad and prominent nasal bridge, globulous nose, downward slanted corners of the mouth, cleft lip and palate, large, low-set ears, clinodactyly, club feet and hypoplastic finger nails. She expired at 11 days after birth. Autopsy revealed an ASD, VSD, and coarctation of the aorta.

Case $1163 / 79$, with a $46, X X, \operatorname{der}(9), t(1 ; 9)(q 41 ; p 24)$ karyotype had the following clinical features: hypertrichosis, hypotelorism, high arched palate, congenital glaucoma with corneal opacity, overlapping fingers and incurved 5th fingers. She expired at the age of 8 months because of bronchopneumonia.

\section{DISCUSSION}

Large scale of chromosome surveys of consecutive liveborn hospital births have been performed using conventionally stained preparations in seven different laboratories (Sergovich et al., 1969; Gerald and Walzer, 1970; Lubs and Ruddle, 1970; Jacobs et al., 1974; Bochkov et al., 1974; Hamerton et al., 1975; Nielsen and Sillesen, 1975). More recently, chromosome surveys of newborn infants utilizing various banding techniques have been published from 3 centers (Lin et al., 1976; Hansteen et al., 1982; Bratkowska et al., 1985). Surveys with banding techniques were restarted in Edinburgh, Scotland (Buckton et al., 1980) and Risskov, Denmark (Nielsen et al., 1982). However, most of these surveys have been performed on largely Caucasian populations, and less was known about Japanese newborn populations. Higurashi et al. (1985) reported data on the birth prevalence of some kinds of chromosome abnormalities among 22,063 newnorn infants in Tokyo, but the initial screening method was somewhat different from that of the present study, as the cases were primarily selected on the basis of their own criteria, and not all infants were chromosomally analyzed.

The purposes of this study are to establish the incidence of major chromosome abnormalities among liveborn infants in Japan and to compare our data with those obtained from the previous surveys of primarily Caucasian infants. During the first period from 1975 to 1983 , chromosome examinations were performed on 10,801 infants using an initial five-cell analysis of conventionally stained preparations. During the second period from 1984 to 1986 , a total of 4,034 infants were chromosomally examined using an initial two-cell analysis of G-banded preparations. 
In Table 7, the frequencies of sex chromosome abnormalities detected in this survey are presented separately, comparing the two periods together with the data obtained from the seven surveys reviewed by Nielsen and Sillesen (1975) and those summarized from three surveys in the literature in which banding analyses were routinely performed (Lin et al., 1976; Buckton et al., 1980; Hansteen et al., 1982). The data for sex chromosome abnormalities from two surveys (Nielsen et al., 1982; Bratkowska et al., 1985) are not included in Table 7, since exact numbers of male and female infants were not given in their reports. Table 8 presents a comparison of the incidence of autosomal abnormalities in our survey, the seven previous surveys without banding, and the five surveys with banding. There was no significant difference in the incidence of any chromosome abnormality between the two periods of this survey. Whether the initial cytogenetic screening was performed on conventionally-stained preparations or on banded preparations, the incidences of major chromosome abnormalities appear to be quite similar.

The incidences of $47, \mathrm{XXY}$ and $47, \mathrm{XYY}$ male infants in this survey were quite close to the mean incidences obtained from seven previous surveys performed without banding. The unusually high frequencies of $47, \mathrm{XXY}$ and $47, \mathrm{XYY}$ male infants were noted in the pooled data from three surveys with banding, but no explanation for this high frequency was found. One female infant in our survey with a $45, X$ karyotype expired at 2 days of life. Only two such 45,X infants were detected among 20,370 females from the seven previous surveys performed without banding. The incidence of $47, \mathrm{XXX}$ females found in this study was quite similar to that ontained from the previous seven surveys performed without banding and that obtained from three surveys with banding. There were eight infants with sex chromosome mosaicism among the first 10,801 infants, but only one among the second 4,034 infants, although the difference was not statistically significant. This may be explained by the difference in the number of cells analyzed during the two periods. The incidence of male infants with abnormal sex chromosomes in this survey was slightly higher than that of female infants. Similar trends were observed in pooled data from the previous seven surveys performed without banding, and those from three surveys with banding.

Balanced Robertsonian and reciprocal translocations occurred with equal frequencies in our series. This observation is in agreement with the previous seven surveys performed without banding. The mean incidence of balanced reciprocal translocations in the pooled data from the five surveys performed with banding was slightly higher than the incidence in this survey and in the seven surveys performed without banding. Although the difference is not significant, the reason for this comparatively higher incidence may be explained by the utilization of the new methods.

Finally, as four pregnancies were electively terminated during this study period after the prenatal detection of abnormal fetal karyotypes, the incidences of major chromosome abnormalities obtained in this survey must be considered to be minimal 
estimates of frequencies in the population.

\section{REFERENCES}

Bochkov, N.P., Kuleshov, N.P., Chebotarev, A.N., Alekhin, V.I. and Midian, S.A. 1974. Population cytogenetic investigation of newborns in Moscow. Humangenetik 22: 139-152.

Bratkowska, W., Hubner, H., Ferenc, T., Szpakowski, M., Krajewski, J. and Budzko, W. 1985. Chromosome studies in 3665 consecutive newborn children. Acta Anthropogenet. 9: 153-161.

Buckton, K.E., O'Riordan, M.L., Ratcliffe, S., Slight, J., Mitchell, M., McBeath, S., Keay, A.J., Barr, D. and Smout, M. 1980. A G-band study of chromosomes in liveborn infants. Ann. Hum. Genet. 43: 227-239.

Gerald, P.S. and Walzer, S. 1970. Chromosome studies of normal newborn infants. In Human Population Cytogenetics. Jacobs, P.A., ed., The University Press, Edinburgh, pp. 143-161.

Hamerton, J.T., Canning, N., Ray, M. and Smith, S. 1975. A cytogenetic survey of 14,069 newborn infants. Clin. Genet. 8: 223-243.

Hansteen, I., Varslot, K., Steen-Johnsen, J. and Langard, S. 1982. Cytogenetic screening of a new-born population. Clin. Genet. 21 : 309-314.

Higurashi, M., Iijima, S., Takeshita, T., Oda, M., Takadaya, K. and Watanabe, N. 1985. Incidence of malformation syndromes and chromosomal abnormalities in 22,063 newborn infants in Tokyo. Jpn. J. Human Genet. 30: 1-8.

Jacobs, P.A., Melville, M., Ratcliffe, S., Keay, A.J. and Syme, J. 1974. A cytogenetic survey of 11,680 newborn infants. Ann. Hum. Genet. 37: 359-376.

Lin, C.C., Gedeon, M.M., Griffth, P., Smink, W.K., Newton, D.R., Wilkie, L. and Sewell, L.M. 1976. Chromosome analysis on 930 consecutive newborn children using quinacrine fluorescent banding technique. Hum. Genet. 31: 315-328.

Lubs, H.A. and Ruddle, F.H. 1970. Applications of quantitative karyotype of chromosome variation in 4400 consecutive newborns. In Human Population Cytogenetics. Jacobs, P.A., ed., The University Press, Edinburgh, pp. 119-142.

Maeda, T., Ohno, M., Takada, M., Kato, Y., Nishida, M., Jobo, T., Adachi, H. and Taguchi, A. 1978. A cytogenetic survey of consecutive liveborn infants-incidence and type of chromosome abnormalities. Jpn. J. Human Genet. 23 : 217-224.

Nielsen, J. and Sillesen, I. 1975. Incidence of chromosome aberrations among 11148 newborn children. Humangenetik 30: 1-12.

Nielsen, J., Wohlert, M., Faaborg-Andersen, J., Hansen, K.B., Hvidman, L., Krag-Olsen, B., Moulvad, I. and Videbech, P. 1982. Incidence of chromosome abnormalities in newborn children. Comparison between incidence in 1969-1974 and 1980-1982 in the same area. Hum. Genet. 61: 98-101.

Sergovich, F., Valentine, G.H., Chen, A.T.L., Kinch, R.A.H. and Smout, M.S. 1969. Chromosome aberrations in 2159 consecutive newborn babies. New Engl. J. Med. 280: 851-855. 